\title{
Alternancia, un cambio de timón que no debiera hacer encallar el barco
}

\author{
Alternation, a change of rudder that should not cause the ship to run \\ aground
}

José Luis SALAS VELÁZQUEZ

Universidad Iberoamericana Puebla

Recibido: 26 de enero del 2019

Aceptado: 24 de febrero 2019

\section{Resumen}

La alternancia en el poder nos enriquece como sociedad. Nos da la certeza de que existen diferentes puntos de vista, diferentes visiones de futuro y de cómo se hacen o cómo debieran hacerse las cosas en lo público. Nos enseña que nadie tiene la verdad absoluta y que el poder no es patrimonio de ningún grupo en particular, de ningún partido, ni de ningún grupo empresarial, sindicato o familia. Nos recuerda que en la política ni los triunfos ni las derrotas serán eternas. Siempre habrá posibilidad de volver, con ideas renovadas y con mayores ímpetus. Este artículo versa sobre la alternancia democrática ventajas y desventajas, desde una mirada de las elecciones mexicanas.

\section{Abstract}

The alternation in power enriches us as a society. It gives us the certainty that there are different points of view, different visions of the future and of how things are done or how things should be done in public. It teaches us that no one has the absolute truth and that power is not the property of any particular group, of any party, or of any business group, union or family. It reminds us that in politics neither triumphs nor defeats will last forever. There will always be the possibility of returning, with renewed ideas and with greater impetus. This article deals with the democratic alternation advantages and disadvantages, from a perspective of the Mexican elections.

Palabras clave: Democracia; Elecciones; Alternancia democrática.

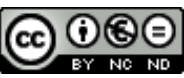

Facultad de Ciencias Sociales y de la Comunicación Universidad de La Laguna Avenida César Manrique, s/n; Campus de Guajara 38071 La Laguna, Tenerife (Islas Canarias - España) 
Keywords: Democracy; Elections; Democratic alternation.

\section{Resultados sin alternancia}

El 11 de noviembre de 2019, en medio de sospechas de fraude electoral e irregularidades identificadas por la Organización de Estados Americanos, Evo Morales, el entonces presidente de Bolivia dimitió a su mandato de casi 14 años en el poder.

Evo Morles, sin duda, es una figura de poder y su trayectoria política es muy interesante. Consiguió ser presidente de Bolivia por primera vez en el año 2006, derivado de un triunfo contundente e inédito que le otorgó entre el $51 \%$ y el $52 \%$ de los votos, con más de 20 puntos por encima de su máximo rival. Una vez en el poder, promovió una serie de cambios políticos, administrativos y jurídicos que tenían por objeto la refundación del Estado Boliviano, mediante una nueva constitución.

El 21 de octubre de 2008, el Congreso de Bolivia aprobó las modificaciones finales de la futura nueva Constitución, que le permitirían reelegirse como presidente de la República. Evo siempre estuvo seguro de que ganaría en las urnas y así fue.

Esta Constitución estableció que sólo podría ser posible buscar la reelección presidencial una vez y, que el mandato de Morales de ese entonces (2006-2010) contaba como su primer periodo. Esto significó que el presidente de Bolivia se podía postular en las elecciones de 2009 y, en caso de vencer, gobernar hasta 2014.

No obstante, en 2013 el Tribunal Constitucional determinó que Evo Morales podría presentarse por tercera vez a una campaña presidencial, algo sin precedentes. La decisión fue declarada como abusiva por la oposición.

No debemos pasar desapercibido que, en 2016 Evo Morales perdió un referendo constitucional con el que pretendía postularse como candidato para un cuarto período en la presidencia. Sin embargo, un fallo judicial le permitió aspirar de nuevo a la presidencia. El 20 de octubre de 2019, Evo Morales ganó de manera controversial un 
cuarto periodo como presidente. Lo que sucedió después es una historia conocida por todos y que hoy se seguirá escribiendo. De lo que no estamos seguros es de cómo terminará.

La gestión de este personaje, lejos de valoraciones ideológicas, es de gran interés. Evo obtuvo logros contundentes, que muchos países en vías de desarrollo quisiéramos constatar. El Producto Interno Bruto (PIB) de Bolivia se incrementó de US\$9.000 millones a US $\$ 40.000$ millones. Además, el crecimiento del PIB per cápita en 2005 fue de 2,5\%, mientras que al cierre de 2018 se ubicó en 2,7\%. La pobreza extrema, se redujo más de la mitad en la última década, pasando de $38 \%$ a $15 \%$. La cifra de desempleo bajó de $8,1 \%$ a 4,2\%. Se aumentó el salario mínimo en los últimos 13 años, pasando de US\$60 a US\$310. También, se inició un proceso intenso de nacionalizaciones, especialmente en el gas, hecho que fortaleció su economía (Bedoya, 2019).

Los resultados obtenidos no fueron suficientes para mantener el poder. No fue suficiente una gestión gubernamental que produjo resultados contundentes en la economía. Ante la tentación de eternizarse en el poder, los grupos de oposición se radicalizaron, los ciudadanos se manifestaron en masa y, de pronto, en un escenario de disturbios sociales, las protestas se generalizan.

La incipiente democracia latinoamericana nos sigue dando lecciones, "la alternancia es indispensable incluso con buenos resultados". Lo cierto es que el poder una vez obtenido por un grupo se ejerce, se mantiene y se quisiera eternizar. La alternancia es fundamental para las democracias verdaderas. Es un componente que evita los cambios abruptos, radicales y destructivos que generan violencia, pobreza y dolor para los pueblos. No obstante, la alternancia no debiera generar retrasos, retrabajos y malos resultados, como ha llegado a suceder. 


\section{Alternancia simulada y resultados}

Empecé este artículo parafraseando las noticias más recientes sobre Bolivia. Sin embargo, para los efectos del mismo, no resulta óbice hacer una breve referencia de la historia político económica de nuestro país. Si bien es cierto en México hemos constatado avances importantes en materia de políticas públicas y algunos resultados concretos, especialmente entre la década de los 50's-60's, el principal logro, fue generar estabilidad más que un progreso generalizado y, sobre todo, de las clases más desprotegidas.

Vivimos más de setenta años de hegemonía partidista, en la que el dominio del Partido Revolucionario Institucional (PRI) fue absoluto. No era posible entender el funcionamiento de la administración pública sin vincularla con la lógica del partido y así nació la noción del llamado "sistema". Todo cabía en el "sistema". Funcionarios de todos los niveles, de todos los ámbitos de gobierno, fueran federales, estatales y municipales. Sindicatos, organizaciones patronales, organizaciones de la sociedad civil e incluso partidos de oposición. Todos estaban más o menos vinculados al partido gobernante. Es muy conocido por todos que este sistema fue llamado por Vargas Llosa como la "dictadura perfecta" (El País, 1990), que llegó a los extremos de permitir, e incluso promover, una alternancia gradual y muchas veces simulada.

El periodo en el que se experimentó más crecimiento, fue denominado como "Desarrollo Estabilizador" y en él "la economía mexicana se vio caracterizada por un alto crecimiento de la producción, bajas tasas de inflación y estabilidad en el tipo de cambio" (Diero en Imágen, 2012). Pareciera entonces que los buenos resultado en la administración pública requirieran de condiciones de estabilidad, continuidad y visión de largo plazo, por sobre avances democráticos y alternancia de los grupos de poder.

\subsection{Alternancia y resultados magros}

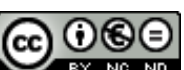

BY NO ND
Facultad de Ciencias Sociales y de la Comunicación

Universidad de La Laguna

Avenida César Manrique, s/n; Campus de Guajara

38071 La Laguna, Tenerife (Islas Canarias - España) 
La hegemonía del PRI vio su fin hasta que llegó a la Presidencia de la República el Partido Acción Nacional (PAN) en el año 2000. Ya en la presidencia de la República, este partido se afianzó, lo que permitió que mantuviera el poder un sexenio más, con Felipe Calderón Hinojosa. No obstante, el escenario político electoral en las entidades federativas y los municipios durante los años que gobernó el PAN se fue tiñendo de varios colores y se fortaleció la alternancia entre los diversos partidos políticos.

Después de doce años en el poder y, a pesar del dominio que generó desde la presidencia, el PAN perdió nuevamente las elecciones a la primera magistratura del país y el PRI regresó al poder. En este sentido, ante el mundo, México demostró el funcionamiento de su sistema democrático, y la posibilidad de una alternancia constante. EI PRI en su regreso, sólo pudo retener el poder un sexenio (el de Enrique Peña Nieto), ya que volvió a perder, en medio de escándalos de corrupción. Ahora es el Movimiento de Regeneración Nacional (MORENA) quien, en representación de la izquierda, gobierna por primera vez, con una amplia aceptación de la población. MORENA, Además, domina el Congreso de la Unión, la mayoría de las gubernaturas estatales y los municipios.

Estos cambios de partidos en el poder, en los diversos ámbitos de gobierno, lamentablemente trajo consigo cambios radicales en las estructuras de gobierno, una gran movilidad de recursos humanos y, lamentablemente, la pérdida de un valioso capital humano que se había ido formando paulatinamente,

\subsection{Las bondades de la alternancia}

La alternancia en el poder nos enriquece como sociedad. Nos da la certeza de que existen diferentes puntos de vista, diferentes visiones de futuro y de cómo se hacen o cómo debieran hacerse las cosas en lo público. Nos enseña que nadie tiene la verdad absoluta y que el poder no es patrimonio de ningún grupo en particular, de ningún 
partido, ni de ningún grupo empresarial, sindicato o familia. Nos recuerda que en la política ni los triunfos ni las derrotas serán eternas. Qué siempre habrá posibilidad de volver, con ideas renovadas y con mayores ímpetus.

La alternancia nunca debe significar el cambio o destrucción de lo bien hecho. No solo por el bien de la ciudadanía que votó para que se produjera, sino porque las sociedades deberían ir construyéndose sobre lo que se ha ido logrando en el pasado.

Mucho daño ha hecho a nuestro país que los gobernantes en turno, en cualquier ámbito de gobierno, quieran reinventar el gobierno federal, los gobiernos estatales o los ayuntamientos cada que hay un nuevo ejercicio gubernamental. Incluso, hemos sido testigos de la cancelación de programas y políticas públicas exitosas. Esto sucede hasta por nuevos gobernantes del mismo partido político, bajo la consigna de que lo que antes se hizo, por definición, está mal hecho.

Pareciera entonces que ¿tenemos que elegir entre alternancia de los grupos de poder o resultados en el gobierno? Estoy convencido de que lograr resultados en la administración pública no debiera de constituir un dilema.

\subsection{Alternancia sin perder el rumbo}

La alternancia en el poder, al generar una sustitución de las más altas posiciones de mando en la administración pública, puede y debe generar nuevas sinergias, mejores acciones y resultados más consistentes. Eso espera la población, eso merecen los votantes. Gobiernos cada vez más eficientes, cada vez más eficaces, cada vez más profesionales.

Para lograr impulsar un proyecto político es necesario que el gobernante cuente con posiciones estratégicas, pero más importante aún es que cuente con un equipo capaz de generar verdaderas políticas públicas y no sólo acciones de gobierno aisladas, ineficiencias, ineficaces y sin sustento.

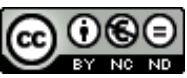

Facultad de Ciencias Sociales y de la Comunicación

Universidad de La Laguna

Avenida César Manrique, s/n; Campus de Guajara

38071 La Laguna, Tenerife (Islas Canarias - España)

www.revistapangea.org | Pág. 19 
Pero ¿cómo lograr un equilibrio entre la innovación, la frescura, los nuevos aires, el sello de los grupos de poder recién llegados y las políticas públicas eficientes, generadas en gobiernos pasados?

Cualquiera que conozca a fondo la administración pública sabe que las necesidades sociales siempre serán mayores que los recursos para atenderlas. Por ello, siempre será retador proponer, diseñar e instrumentar desde las dependencias y entidades públicas soluciones que busquen los mejores y mayores resultados con los recursos humanos, técnicos, financieros y materiales que se tienen disponibles.

Articular un programa efectivo en la administración pública no debería ser resultado de una ocurrencia. Sí es posible que parta de una buena idea, pero requiere de muchos otros elementos. Un diagnóstico adecuado, una investigación seria que considere datos de instituciones especializadas y experiencias o mejores prácticas de gobierno. Se requiere de un diseño adecuado, del análisis de las variables y de las externalidades que puedan afectar la propuesta. Aunado a ello, se necesita una adecuada planeación presupuestal, validación técnica, jurídica, social e incluso política. Encontrar soluciones apropiadas para los problemas sociales nos exige identificar los mecanismos más adecuados para lograr su desarrollo, un seguimiento puntual de los avances y resultados, una medición objetiva, una evaluación integral y, por supuesto, de la voluntad política para impulsar la propuesta.

Quien conozca del servicio público, sabrá que el ejercicio de la función pública requiere el dominio de conocimientos especializados y diversos que permitan la viabilidad de las propuestas de gobierno.

Por todo lo anterior, las nuevas ideas y la innovación de la gerencia gubernamental debe ir acompañada de la técnica y de la experiencia. Esto sólo es posible si contamos con servidores públicos calificados, experimentados y con los conocimientos suficientes para conducir a buen camino las propuestas. 


\section{Profesionalización, servicio civil de carrera y corrupción}

Es justo aquí donde radica el quid del asunto que abordamos en este artículo. Tanto la profesionalización de los servidores públicos del Estado, como la implementación de un servicio civil de carrera no son, de ninguna manera, temas nuevos. Son varios los esfuerzos que se han hecho por impulsar mecanismos que permitan la selección de los mejores perfiles, la capacitación del personal que ya labora en la administración pública e incluso la formación de funcionarios con las capacidades técnicas que requiere la función pública.

Lamentablemente, todos los esfuerzos de instituir un verdadero servicio civil de carrera en las administraciones públicas de nuestro país han sido limitados, parciales, con poca visión y, tal vez, con menos voluntad política. Pareciera que nos resistimos a perder ese gran coto de poder que significa la posibilidad de seleccionar a la mayoría de los servidores públicos de nivel directivo o decisorio que acompañan al grupo en el poder e, incluso, hemos sido testigos de cambios bruscos en todos los niveles de decisión, a pesar de que se comprometa la adecuada operación de los programas.

La falta de implementación de un servicio civil de carrera afecta la calidad de las decisiones públicas y sus resultados. No sólo es un tema de eficiencia y eficacia, de hecho, para algunos autores, esta posibilidad constituye un área de oportunidad para que germine la corrupción. El desplazamiento de la mayoría del personal de una administración pública puede producir algo mucho más complejo y grave, que algunos autores han llamado "la captura del Estado y de los puestos públicos".

En este sentido, el Doctor Mauricio Merino del Centro de Investigación y Docencia Económicas, CIDE (Merino, 2013) nos ha explicado con gran claridad este punto que establece una relación causal entre la corrupción y la inexistencia o ineficacia de un 
verdadero servicio civil de carreta en la administración pública. Par entender esta tesis, brevemente esbozaremos algunas ideas.

No queda duda de que la corrupción es la causa de la ineficiencia gubernativa y del desencanto democrático. Hemos identificado a la corrupción como la causa de inequidad, de la injusticia y de otros tantos males sociales. No obstante, nos hemos quedado cortos al analizar ¿cuál es la causa de la corrupción? Entre estas causas (y otras más, que no analizaremos en este artículo) encontramos la captura de los puestos públicos.

Tradicionalmente se ha entendido la corrupción desde dos visiones, una individualista y otra burocrática. Según el primer enfoque los sistemas son inmunes a la corrupción y son los individuos los que los corrompen. Con esta lógica se buscan soluciones éticas y moralizantes que promueven los valores y las buenas conductas de los servidores públicos y dichas conductas son valoradas desde un grupo de poder. Como consecuencia, este grupo de poder impulsa la salida y eliminación de aquellas personas que no comparten sus principios, su ideología e incluso hasta su partido político. "Si cambiamos a los individuos corrompidos, se acaba la corrupción”. Este enfoque si bien tiene lógica y, en muchos casos, sustento, no debe tomarse a la ligera.

La administración entrante tiene el deber de identificar a los servidores públicos eficientes y aprovechar sus capacidades y experiencia. A la par, se puede iniciar un proceso paulatino de expulsión de servidores públicos involucrados en actos de corrupción que debería estar basado en la comprobación de responsabilidades administrativas y no sólo por la falta de pertenencia a un grupo político.

Las consecuencias de este enfoque individualista que pugna por la renovación "automática e indiscriminada" de las cuotas de poder con base en la lealtad, en la pertenencia a un grupo y en la ubicación de los amigos, genera una nueva forma de captura, ahora justificada con nuevos argumentos. Esta visión no sólo es limitada, sino 
perversa porque tiene como resultado un control de puestos clave que, en corto o mediano plazo, vuelven a ser susceptibles de actos de corrupción.

El segundo enfoque (burocrático) propone concentrar los esfuerzos anticorrupción a través de sus efectos burocráticos. Es decir, sobre las acciones correctivas y las sanciones que deben aplicarse sobre hechos consumados y, en algunos casos, buscar la reparación del daño causado. Esto no significa que la impunidad de los actos corruptos no sean un gran incentivo para cometerlos y que se deban generar acciones concretas para mejorar los sistemas de investigación y sanción de las conductas corruptas. Si bien se trata de un enfoque más objetivo, su debilidad radica en que se asume la congruencia, neutralidad e idoneidad del marco legal. O sea, este enfoque concentra sus esfuerzos en el individuo (servidor público-empresa) que comete corrupción violentando las reglas y los protocolos establecidos. Incluso persigue a aquellos que, sin cometer un acto corrupto, no se sometieron a las reglas establecidas, aún en el caso en el que no hayan obtenido un beneficio privado. Lo que afirma que la corrupción es una anomalía o distorsión del sistema que sería perfecto si los individuos se condujeran de acuerdo a las reglas. Así las cosas, quedan fuera los actos de corrupción que se realizan con una apariencia de legalidad y cumpliendo las normas y los protocolos. Adicionalmente, debe producirse evidencia suficiente para que se pueda sancionar a los individuos corruptos. Se castiga al que se equivoca.

Siguiendo las ideas del Doctor Merino, creemos que estas reflexiones no significan que los enfoques explicados propongan acciones innecesarias. Al contrario, la dimensión de la corrupción en nuestro país hace necesarias estas acciones, pero la corrupción debe analizarse como un fenómeno sistémico, producto de la captura de los puestos o posiciones gubernamentales, de la captura de las decisiones públicas y de la captura de los presupuestos públicos. Ante estos supuestos, un verdadero servicio civil de carrera podía constituir un factor de contención. 
Mientras los grupos de poder decidan, sin limitaciones jurídicas y administrativas, quiénes ocupan los puestos públicos y esas decisones se tomen bajo criterios de lealtad; mientras controlen las decisiones públicas y la distribución de recursos, será casi imposible encontrar soluciones adecuadas a la corrupción. A este conjunto de factores los autores le han llamado "La tragedia de lo público" y parte de esa tragedia la constituyen algunas decisiones que la alternancia del poder genera cuando no toman en cuenta la opinión, la experiencia y la técnica de las mujeres y hombres que conforman la administración pública

\section{La profesionalización de los servidores públicos como una alternativa para el Estado de Puebla}

El pasado proceso electoral en Puebla hizo posible el primer gobierno de izquierda en el Estado. La alternancia de los grupos en el poder generó en la población altas expectativas. Habrá que hacer las cosas bien, con nuevas visiones, con nuevas orientaciones y dar resultados. No obstante, habrá que ser cuidadosos para que los cambios de timón nos lleven a buen puerto y no exista la posibilidad de encallar el barco por falta de experiencia. Es un gran reto, pero se tiene que asumir y para lograrlo, deberá encontrarse esa muy fina combinación entre el entusiasmo, las buenas ideas, las ganas de innovar y la experiencia basada en el conocimiento técnico.

Hay un gran potencial en los recursos humanos del servicio público y habrá que aprovecharlo.

Hoy el Gobierno del Estado de Puebla tendrá en sus manos la posibilidad de instrumentar un mecanismo serio de profesionalización, capacitación y permanencia de los servidores públicos que construya un verdadero servicio civil de carrera. Un proyecto necesario y, que, si se instrumenta adecuadamente, marcaría un hito en la administración pública estatal y contribuirá a aminorar la corrupción.

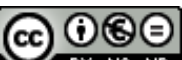

EY NC ND
Facultad de Ciencias Sociales y de la Comunicación

Universidad de La Laguna

Avenida César Manrique, s/n; Campus de Guajara

38071 La Laguna, Tenerife (Islas Canarias - España) 
El reto no es menor, porque para que esto pueda ser adecuadamente implementado deberá considerar al menos las siguientes variables:

- Un diagnóstico serio de mejores prácticas en materia de implementación de servicios civiles de carrera nacionales e internacionales que identifique los aciertos y las áreas de oportunidad para su perfeccionamiento;

- Un diseño adecuado que abarque los mecanismos de ingreso, permanencia, capacitación, evaluación y seguimiento de los servidores públicos que se inscriban.

- Una evaluación seria de los logros y áreas de oportunidad del servicio civil de carrera que se empezó a implementar en la administración pública estatal, pero que requiere un reforzamiento sin precedentes.

- Que se capacite y evalúe al servidor público, no sólo en aspectos técnicos, sino en "soft skills", tan necesarias en el día a día de la actividad gubernamental (inteligencia emocional, atención al público, resolución de conflictos, manejo del estrés, trabajo colaborativo, entre otras)

- Mecanismos que mantengan el interés del servidor público de carrera por una capacitación continua y para seguir dando lo mejor de sí.

- Una legislación adecuada para instituir e implementar el servicio civil de carrera.

- Un organismo o conjunto de instituciones públicas y privadas que cuiden de la adecuada implementación del servicio civil de carrera.

Con la instrumentación de un adecuado servicio civil de carrera en el Estado de Puebla, se daría un paso firme hacia la eficiencia, la eficacia y el combate a la corrupción.

\section{Conclusiones}

- La alternancia del poder es fundamental para la vida democrática de las sociedades. Imprime dinamismo en la administración pública y debe promover soluciones innovadoras a los problemas y necesidades sociales.

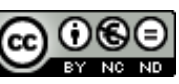

Facultad de Ciencias Sociales y de la Comunicación

Universidad de La Laguna

Avenida César Manrique, s/n; Campus de Guajara

38071 La Laguna, Tenerife (Islas Canarias - España) 
- La alternancia democrática incluye la llegada de nuevos grupos de poder, que requieren la designación de funcionarios en posiciones estratégicas que permitan hacer realidad su visión, sus planes de gobierno y sus compromisos de campaña.

- La designación de los funcionarios clave que representan los propósitos de los nuevos grupos de poder debe considerar sus aptitudes, su capacidad, su experiencia e, incluso, considerar a las mujeres y hombres que ya forman parte del servicio público, ya que el reto al que se enfrentarán no es menor.

- Siempre serán más los problemas sociales y necesidades públicas que requieren atención a los recursos financieros, materiales y humanos con los que se disponen. Por ello, la administración pública tiene la responsabilidad de que cada programa gubernamental genere el mayor y mejor resultado posible.

- Las soluciones de política pública requieren conocimientos, técnica y experiencia. En este sentido, son necesarios los equilibrios entre los nuevos funcionarios y los servidores públicos de administraciones públicas pasadas.

- Los funcionarios en activo deben de profesionalizarse, capacitarse y aportar lo mejor de sí mismos, viendo la alternancia del poder como una oportunidad de hacer mejor las cosas, de transformar las viejas prácticas y atreverse a innovar.

- La profesionalización de los servidores públicos debe ir acompañada de un Servicio Civil de Carrera que otorgue estabilidad a las mujeres y hombres que apuestan su desarrollo profesional a la administración pública.

- Hacer realidad un servicio civil de carrera contribuirá a disminuir la corrupción, aumentar la eficiencia de las acciones públicas y hacer de la alternancia del poder una oportunidad de mejorar.

\section{Bibliografía}


Bedoya, M. P. (23 de octubre de 2019). Los cinco logros económicos de los 13 años de gestión de Evo Morales en Bolivia. LR La República. (L. L. República, Ed.) Bogotá, Colombia. Recuperado el noviembre de 2019

Dinero en Imágen. (26 de 12 de 2012). ¿Qué fue el Desarrollo Estabilizador?: Paradigmas. CDMX, México. Obtenido de https://www.dineroenimagen.com/2012-1226/13356

El País. (01 de Septiembre de 1990). Vargas Llosa: "México es la dictadura perfecta". Obtenido de elpais.com: https://elpais.com/diario/1990/09/01/cultura/652140001_850215.html

Merino, M. (10 de Julio de 2013). La Captura de los puestos públicos. Revista Mexicana de Ciencias Políticas y Sociales, Nueva Época, Año LVIII, ISSN-0185-1918(219), 135. Recuperado el 25 de noviembre de 2019, de file:///C:/Users/DT1x/Google\%20Drive/1.1\%20CACEP/Documentos\%20de\%20Consu Ita/Publicaciones/La\%20captura\%20de\%20los\%20puestos\%20p\%C3\%BAblicos,\%20 Merino\%20Mauricio.pdf

\section{Forma de citar este artículo en bibliografías}

SALAS VELÁZQUEZ, José Luis (2019): "Alternancia, un cambio de timón que no debiera hacer encallar el barco", en Revista PANGEA № 10, páginas 14 a 27. Tenerife: Red Académica Iberoamericana de Comunicación. Recuperado el _ de de 2 de: http://www.revistapangea.org 